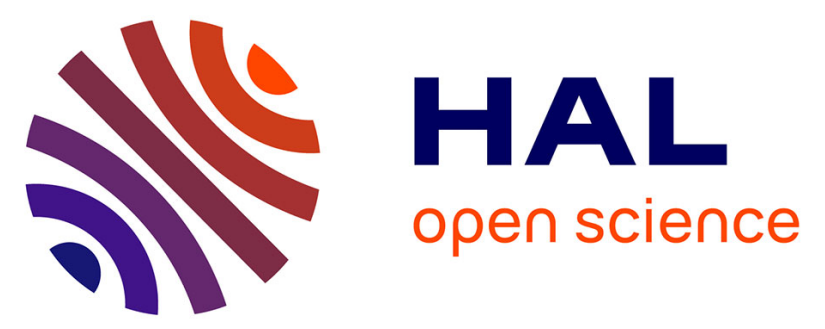

\title{
Conduite à tenir pour le personnel soignant en contact avec des patients atteints de diphtérie
}

\author{
Joana Brousse, R. Cruchet, Marie-Agnès Denis, L. Hees, J. Grando, P. \\ Girardo, P. Primat, D. Floret, K. Tarabit, Philippe Vanhems, et al.
}

\section{- To cite this version:}

Joana Brousse, R. Cruchet, Marie-Agnès Denis, L. Hees, J. Grando, et al.. Conduite à tenir pour le personnel soignant en contact avec des patients atteints de diphtérie. Archives des Maladies Professionnelles et de L'Environnement, 2018, 80 (1), pp. 58-66. 10.1016/j.admp.2018.10.005 . hal-02089379

\section{HAL Id: hal-02089379 \\ https://hal.science/hal-02089379}

Submitted on 21 Oct 2021

HAL is a multi-disciplinary open access archive for the deposit and dissemination of scientific research documents, whether they are published or not. The documents may come from teaching and research institutions in France or abroad, or from public or private research centers.
L'archive ouverte pluridisciplinaire HAL, est destinée au dépôt et à la diffusion de documents scientifiques de niveau recherche, publiés ou non, émanant des établissements d'enseignement et de recherche français ou étrangers, des laboratoires publics ou privés.

\section{(ㄷ)(1) $\$$}

Distributed under a Creative Commons Attribution - NonCommerciall 4.0 International 
Version of Record: https://www.sciencedirect.com/science/article/pii/S1775878518307392

\title{
Conduite à tenir pour le personnel soignant
}

\section{en contact avec des patients atteints de}

\author{
diphtérie
}




\section{Summary :}

Introduction : The Occupational health Service has implemented a Simplified Code of Conduct based on the High Council of Public Health 'guide dated March 4th 2011 in case medical staff comes in contact with a patient suffering from diphtheria, ORL or cutaneous. This article describes the hospitalization of two children suspected of cutaneous diphtheria and the support provided to the staff.

Material and method: The approach was to list the caregivers without protection while in contact with the children to set up a clinical surveillance, to do nose and throat swabbing, to give a prophylactic antibiotic treatment regardless of the immunization status and the result of the culture, and perform a diphtheria booster if the last was more than 5 years.

Results : We identified 64 caregivers in contact. 43 were sampled, 31 received a prophylactic antibiotic treatment and 9 a vaccination reminder. Three days after the alert, the two children's skin samples came back negative, but the pharyngeal swabs positive for Corynebacterium diphteriae $(C d)$. On the $6^{\text {th }}$ day we learned that $C d$ isolates found in the children's throats did not carry the tox gene, coding for diphtheria toxin (tox-) and on the $11^{\text {th }}$ day we decided to stop our approach. This simplified Code of Conduct is presented as a flow chart.

Conclusion: This investigation follows a 34 caregivers' screening performed in an Adults' Hospital. This staff was in contact with the wounds of the sister of our two patients infected with an isolate of $C d$ carrier of the tox gene (tox +$)$. This same Hospital has had to manage two other cases of cutaneous diphtheria (tox+), then oropharyngeal (tox-) without secondary case identified.These unexpected episodes must remind everyone of the importance of the respect for immunization schedules and of the standard and additional precautions to be taken.

Keywords: occupational medicine, occupational health services, health care worker, diphtheria, epidemics // 


\section{Résumé :}

Introduction : Le Service de Santé au Travail (SST) a mis en place une Conduite à tenir simplifiée (CATS) basée sur le guide du Haut Conseil de la Santé Publique du 4 Mars 2011, en cas de contact du personnel avec un patient atteint de diphtérie ORL ou cutanée. L'objectif de cet article est de décrire l'hospitalisation de 2 enfants suspects de diphtérie cutanée et la prise en charge pour les soignants.

Matériel et Méthodes : La démarche a consisté à lister les professionnels contacts sans protection lors des passages des enfants pour instituer une surveillance clinique, réaliser des écouvillonnages nasaux et pharyngés, délivrer une antibioprophylaxie (ABP) indépendamment du statut vaccinal et du résultat de la culture, et effectuer un rappel antidiphtérique si le dernier datait de plus de 5ans.

Résultats : Nous avons identifié 64 agents contacts. Quarante trois ont eu des prélèvements, 31 ont reçu une ABP et 9 un rappel vaccinal. Trois jours après l'alerte, les prélèvements cutanés des 2 enfants sont revenus négatifs mais les écouvillonnages oropharyngés positifs à Corynebacterium diphtheriae $(C d)$. Au $6^{\text {ème }}$ jour nous avons appris que les isolats de $C d$ retrouvées dans les gorges des enfants n'étaient pas porteurs du gène tox, codant pour la toxine diphtérique (tox-) et au $11^{\text {ème }}$ jour, nous avons arrêté notre démarche. Cette CATS est présentée sous forme de logigramme.

Conclusion: Cette enquête fait suite à un dépistage dans l'hôpital voisin de 34 soignants contacts avec les plaies de la sœur de nos deux patients, infectée par un isolat de $C d$ porteur du gène tox (tox+). Ce même hôpital a eu à gérer 2 autres cas de diphtérie cutanée (tox+) puis oropharyngée (tox-) sans cas secondaire identifié. Ces épisodes inattendus rappellent l'importance du respect du calendrier vaccinal et des précautions standard et complémentaires.

Mots clefs: médecine du travail, service de santé au travail, personnel soignant, diphtérie, épidémie, logigramme 


\section{Cas clinique}

En septembre 2016, 2 frères sénégalais de retour avec leur grande sœur d'un voyage familial au Sénégal sont hospitalisés au sein d'un grand CHU français de plus de 5000 lits.

Leur grande sœur Mme Z., âgée de 21 ans, est drépanocytaire et est hospitalisée, une semaine après son retour en France, dans le service de Médecine Interne de l'hôpital pour adultes voisin pour une crise vaso-occlusive accompagnée de lésions authentifiées comme une diphtérie cutanée : lésions prurigineuses diffuses surinfectées des membres inférieurs. Elle est à jour de sa vaccination Diphtérie Tétanos Poliomyélite (DTP).

Le plus grand des frères, I., a 14 ans, est drépanocytaire et également à jour de sa vaccination DTP. Il a consulté auparavant pour des crises vaso-occlusives, donc sans mesure d'isolement par rapport à un risque diphtérique, au sein de l'hôpital pédiatrique, une première fois le jour même de son retour du Sénégal, puis 4 jours plus tard pour seconde crise multifocale avec passage au Service d'Accueil des Urgences (SAU), transfert dans l'Unité d'Hospitalisation de Courte Durée (UHCD) puis une hospitalisation de deux jours dans un centre spécialisé en hématologie pédiatrique. Durant ses hospitalisations pour crises vaso-occlusives, il présente des lésions cutanées des jambes prédominantes au niveau de la jambe droite, érythémateuses avec collerette périphérique, et aucun signe d'infection oropharyngée.

Son petit frère, Y., 7 ans, sans antécédent, a un retard de 6 mois dans sa vaccination DTP. Il est hospitalisé et isolé en même temps que son frère, deux jours après la découverte des résultats des prélèvements cutanés de leur sœur. Il présente des lésions cutanées des membres inférieurs et sur le pavillon de l'oreille droite, sans aucune atteinte ORL.

Dès cette nouvelle hospitalisation, les soignants sont alors informés d'un possible contage avec un cas de diphtérie, la grande sœur pourrait être considérée comme le cas source à partir 
duquel se seraient contaminés les deux enfants. Les soignants ont mis en place un isolement contact et gouttelettes (mise en chambre individuelle, port d'un masque chirurgical, de surblouses, gants à usage unique et hygiène des mains avec une solution hydro-alcoolique). Des prélèvements cutanés et des écouvillonnages nasaux et pharyngés ont été réalisés chez les deux patients. La recherche toxinique est réalisée au Centre National de Référence (CNR) à l'Institut Pasteur à Paris.

Le bactériologiste référent informe le service de Santé au travail (SST) le jour même du lancement de l'alerte, que la souche isolée dans les plaies de la grande sœur est un Corynebacterium diphtheriae $(C d)$ tox + et que ses prélèvements de gorge sont négatifs.

Les résultats des petits frères reviennent trois jours plus tard révélant des prélèvements des lésions cutanées négatifs mais des écouvillonnages oropharyngés positifs à $C d$. L'historique du cas clinique et des contacts est récapitulé en figure 1. Un signalement auprès de l'ARS est alors réalisé par le service hospitalier.

La question qui se pose ici est qu'elle est la conduite à tenir en matière de prise en charge des soignants vis-à-vis de ce risque de contamination diphtérique et le rôle du SST dans cette démarche?

\section{$\underline{\text { Commentaires }}$}

Au mois de septembre 2016, le Service de médecine et Santé au Travail (SST) a été confronté à une situation rare de contage diphtérique.

\section{I. $\quad \underline{\text { Rappels }}$}


La diphtérie est engendrée par différentes espèces de Corynébactéries du complexe diphtheriae: - principalement Corynebacterium diphtheriae $(C d)$, hautement contagieux, qui présente une transmission inter-humaine par contact direct avec des secrétions rhinopharyngées, les gouttelettes de Flügge, ou des plaies cutanées et très rarement par contact indirect avec des objets souillés par des secrétions de malades ; - Corynebacterium ulcerans, reconnu il y a quelques années comme pouvant être transmis par des animaux de compagnie tels que les chiens et les chats; - Corynebacterium pseudotuberculosis, cette dernière espèce étant surtout rencontrée chez les personnes exposées aux ovins ou caprins. Pour ces deux dernières espèces, la transmission inter humaine n'a jamais été observée (1).

La diphtérie présente une période d'incubation de 2 à 5 jours, avec une contagiosité possible de deux semaines à plus d'un mois pour un portage sain. Les symptômes observés sont soit respiratoires avec une angine blanche à pseudo-membranes, une fièvre modérée aux alentours de $38,5^{\circ} \mathrm{C}$, une pâleur et de possibles adénopathies sous-maxillaires, pouvant évoluer vers une détresse respiratoire par atteinte laryngée appelée le croup diphtérique, soit cutanés avec présence de plaies ou d'ulcérations souvent poly-contaminées sur lesquelles peuvent également s'observer des fausses membranes (2).

La gravité de la pathologie est liée à la faculté de certains isolats, dits toxinogènes, à produire la toxine diphtérique (pour cela la bactérie doit posséder le gène tox qui code la toxine et doit la sécréter $($ tox+)). Dans le cas d'une infection toxinique, uniquement possible de nos jours en cas d'absence ou de retard de rappel vaccinal, d'autres manifestations cliniques peuvent être observées participant à la gravité de la maladie, telles que la myocardite, des névrites, des lésions rénales ou hépatiques, une paralysie du diaphragme et une atteinte du système nerveux central pouvant amener à la mort par arrêt cardio-respiratoire (2). 
La vaccination contre la diphtérie est une vaccination anti-toxinique (3), (4) c'est à dire un vaccin préparé en modifiant la toxine diphtérique de façon à ce qu'elle garde ses propriétés immunologiques en perdant son caractère pathogène. Ainsi, les anticorps produits vont inactiver la toxine diphtérique mais n'agissent pas sur la bactérie elle-même, qui peut donc continuer à circuler. Même si le schéma vaccinal est bien respecté, il protège de la maladie mais il permet quand même un hébergement et une transmission des souches toxinogènes.

La vaccination antidiphtérique est obligatoire en France depuis 1938 (5). Cette vaccination, associée aux vaccins antitétanique et anticoquelucheux (DTC) a fait partie du Programme élargi de vaccination de l'OMS depuis sa création en 1974. Notons qu'au cours des années 1980 à 2000, le nombre total de cas de diphtérie notifiés dans le monde a chuté de plus de $90 \%$ (3) et qu'aujourd'hui, la diphtérie est une affection quasiment disparue en France métropolitaine (6). Le dernier cas autochtone à Corynebacterium diphtheriae $(C d)$ déclaré dans l'hexagone date de 1989 (1). Cependant elle persiste dans différents pays et reste un sujet de préoccupation sur différents points car d'une part son importation est toujours possible à partir de pays endémiques et d'autre part, on ne peut agir sur la circulation de Corynebacterieum ulcerans de réservoir animal.

La diphtérie est une maladie à déclaration obligatoire auprès de l'Agence Régionale de Santé (ARS) depuis le 27 mai 2003 (7).

\section{Conduite à tenir en cas de contage diphtérique}

Il n'existe aujourd'hui que très peu d'études épidémiologiques et d'hygiène concernant la diphtérie. Le protocole du Haut Conseil de Santé Publique (HCSP) de 2011 (1), à disposition des cliniciens, a permis dans ce contexte et au vu de son inexistence dans la bibliographie, la rédaction par l'équipe du SST d'une Conduite à tenir simplifiée (CATS). L'objectif de cet 
article est de décrire l'histoire ayant mené à l'hospitalisation des enfants et ayant enclenché la prise en charge des soignants contacts puis de présenter cette CATS sous forme de logigramme exposé en figure 2, afin d'apporter une aide facilement et rapidement applicable pour tous les SST qui pourraient se trouver confrontés à une telle situation.

\section{A. Mise en place de la CAT}

Immédiatement et sans attendre l'arrivée des résultats du laboratoire, nous commençons l'élaboration d'une Conduite à tenir simplifiée (CATS) en nous appuyant sur les recommandations du guide du HCSP du 4 Mars 2011 (1). Selon les recommandations, si la souche est tox + , la recherche de tous les cas contacts doit commencer alors que si elle est tox-, le traitement ne concernera que le cas source avec une recherche de cas symptomatiques dans son entourage familial uniquement et réalisation de prélèvements si atteinte ORL ou lésion cutanée évocatrice. Ceci signifierait l'arrêt de la procédure en SST.

N'ayant pas encore ce résultat toxinique mais au vu du résultat microbiologique de la sœur et du risque élevé de possible transmission aux deux frères, nous avons considéré que ces derniers pouvaient être porteurs asymptomatiques d'une souche tox+.

La démarche, selon les recommandations (1) face à un portage ORL de $C d$ tox+, consiste à considérer le porteur asymptomatique comme un cas et donc de :

- Lister les professionnels contacts sans protection gouttelettes par masque chirurgical lors des différents passages des enfants dans l'hôpital.

- Rechercher des signes cliniques de diphtérie ORL et cutanée chez les professionnels contacts. 
- Réaliser de manière systématique des écouvillonnages nasaux et pharyngés à tous les contacts pour recherche de la bactérie.

- Délivrer une antibioprophylaxie (ABP) par Amoxicilline pendant 7 jours (3g/j en 3 prises) quel que soit le statut vaccinal et le résultat de la culture (ou par Macrolide si allergie : Azithromycine 3 jours, 500mg/j en une prise), au vu du risque de portage et de transmission (contagiosité pouvant durer jusqu'à un mois après le contage) (2). L'ABP est nécessaire pour rompre la chaîne de transmission.

- Effectuer un rappel de vaccination antidiphtérique si le dernier rappel date de plus de 5 ans.

Notre action se met rapidement en place avec contact par courriel puis par appel téléphonique des cadres des unités de service concernés (Service d'Accueil des Urgences (SAU), Unité d'Hospitalisation de Courte Durée (UHCD), Brancardage, Radiologie et le centre hématologique) afin d'obtenir le listing des personnels présents à chaque passage des enfants. Nous lançons une information générale à tous les cadres, cadres supérieurs et médecins référents des services afin de leur expliquer notre démarche.

Nous recommandons au début du traitement antibio-prophylactique ou en attendant sa prescription le port d'un masque chirurgical pour les soignants ayant pu être en contact avec les enfants. Le personnel est averti que toute manifestation clinique à type de dysphagie avec angine fébrile ou de lésion cutanée devra faire l'objet d'une consultation en urgence auprès d'un médecin.

Nous faisons valider notre démarche par le Président du Comité technique des vaccinations du HCSP, professeur émérite de pédiatrie et ancien consultant hospitalo-universitaire en infectiologie pédiatrique. Nous travaillons en collaboration avec les médecins pédiatres 
urgentistes et leurs cadres de santé, ainsi qu'avec le médecin de veille sanitaire de l'Agence Régionale de Santé (ARS) de notre région.

\section{B. Résultats}

\section{Investigations concernant les contacts}

Les consultations médicales débutent le lendemain de l'annonce de la découverte de l'isolat de $C d$ dans les prélèvements ORL des 2 patients, soit le $4^{\text {ème }}$ jour après le lancement de l'alerte. Il est mis en place un isolement gouttelettes dès l'arrivée dans le SST : les agents portent des masques chirurgicaux et se désinfectent les mains en arrivant. Les prélèvements nasaux et pharyngés sont réalisés sur écouvillons secs avec une protection par masque chirurgical, les antibiotiques sont prescrits et les rappels vaccinaux (vaccin dTPCa : Diphtérie Tétanos Poliomyélite et Coqueluche) faits au besoin.

Au total, 78 agents tous métiers confondus ont été contactés, soit : 25 infirmiers (IDE) dont un étudiant IDE, 12 auxiliaires de puériculture (AP) dont également un étudiant AP, 9 médecins, 2 internes, 1 faisant fonction d'interne, ainsi que 9 externes étudiants en médecine, 6 brancardiers, 6 aides-soignants, 5 secrétaires médicales et 3 manipulateurs en radiologie.

Des relances ont du être réalisées afin d'obtenir la liste du personnel médical présent (médecins et étudiants) et également du personnel administratif. Certains agents ont aussi parfois contacté directement le SST.

Sur les 78 agents : 14 indiqués par leur hiérarchie, n'avaient pas réellement été en contact avec les deux enfants. Soixante-quatre ont donc été définis comme sujets contacts, et parmi eux 15 n'ont pas répondu. Quarante neuf personnels de l'hôpital ont donc donné suite à l'alerte, soit 76,6\% des sujets définis comme contacts. 
Sur ces 49: 2 médecins et 1 interne ont dit avoir pris toutes les précautions nécessaires en isolement contact et gouttelettes, 3 médecins et 2 internes ont été entièrement pris en charge dans un autre centre, les 41 personnes restantes ont bien eu des écouvillonnages nasaux et pharyngés en SST.

Trente huit ont été prélevées dans le SST du CHU dont deux agents qui présentaient une forte fièvre avec angine depuis plus de 48 h qui ont été redirigés directement vers les urgences avec le protocole adéquat et prélevés par des urgentistes (prélèvements qui malheureusement dans les 2 cas ont finalement dû être refaits pour vice de procédure et mauvais conditionnement, ce qui montre le manque de connaissances actualisées face à une telle situation) et qui ont reçu une antibiothérapie pour eux et leur entourage proche symptomatique (un mari et une fille de 2ans), un agent a été prélevé directement dans son service et 2 agents du transport externe ont été adressés à leur hôpital de ralliement.

Sur ces 39 personnes prélevées dans l'hôpital pédiatrique, 38 ont donc été reçues en visite médicale avec surveillance clinique, seulement 29 ABP ont finalement été prescrites après interrogatoire plus poussé sur les conditions exactes de contage (soit 74,4\%) et 9 rappels vaccinaux ont été réalisés pour des agents dont le dernier rappel datait de plus de 5 ans (soit $23 \%)$.

Un flow chart de la population est présenté en figure 3 et le tableau descriptif des agents prélevés en figure 4.

\section{$\underline{\text { Résultats microbiologiques }}$}


Concernant les cas index ( 2 frères), le laboratoire de microbiologie a annoncé au service dès le lendemain du lancement de la démarche que la souche de $C d$ était bien sensible à l'amoxicilline et aux macrolides.

Deux jours plus tard, le SST est averti que les 2 souches de $C d$ isolées des gorges d'I. et Y. ne portent pas le gène tox dans leur matériel génétique. Nous aurons alors un contact avec le médecin de l'ARS de notre région, qui nous conseillera, bien que les résultats aient conclu à des isolats tox-, de poursuivre la même attitude chez tous les contacts restants avec écouvillonnages et $\mathrm{ABP}$ systématiques au vu de la possibilité d'un co-portage de souches tox+ et tox-, voir d'une erreur du laboratoire.

Du fait des difficultés d'obtention du milieu de culture adéquat (Milieu de Tinsdale), les résultats des prélèvements nasaux et pharyngés des agents sont communiqués la semaine suivante. Tous reviennent négatifs (un échantillon était non valide et n'a pas pu être recontrôlé), permettant la réassurance des agents.

\section{Finalisation de la CATS}

$\mathrm{Au} 11^{\text {ème }}$ jour après l'alerte, soit trois semaines après les derniers contacts, et après avis pluridisciplinaire, la démarche est finalisée.

Pour information, le cas de la sœur des deux patients, Mme Z., a engendré pour le SST de son lieu d'hospitalisation (l'hôpital pour adulte à proximité), la prise en charge de 34 soignants ayant pu être en contact avec ses plaies (CAT envers un isolat de $C d$ tox+ sans atteinte ORL), soit la réalisation de 32 écouvillonnages, la prescription de 29 ABP et d'un rappel vaccinal.

Enfin, nous avons adapté notre CATS sous forme de logigramme, présenté en figure 2. 


\section{Discussion}

Dans ce cas clinique, l'attitude adoptée est d'une prudence maximale car il a été décidé de rechercher tous les agents ayant pu être en contact avec les enfants lors de leurs différents passages dans l'hôpital sans respecter la limite de 7 jours précédant le contact, ceci en lien avec le temps d'incubation de la maladie qui est de 2 à 5 jours. Cette limite n'a pas été respectée au vu du risque pour les soignants, non pas de développer la pathologie, mais de portage sain et de contagiosité qui peut alors perdurer plus d'un mois après le contage (2). Cette décision a été prise, par précaution, notamment à l'égard des soignants travaillant en centre d'hémato-oncologie pédiatrique et côtoyant des enfants très fragiles.

La décision a du être prise très rapidement d'envisager une transmission de la souche tox+ de la sœur à ses deux frères et de continuer la démarche devant un isolat de Corynebacterium diphtheriae qui s'est finalement avéré tox-. Cependant, cette attitude qui s'est avérée trop prudente, pouvait se justifier au vu des conséquences dramatiques qu'un isolat tox+ aurait pu engendrer et alors que cette hypothèse paraissait alors la plus probable. Le résultat toxinique de la souche des deux frères a été rendu 3 jours après le début du lancement de la démarche en SST, soit au cours de la troisième journée de visites médicales, alors que la majorité des agents définis comme contacts avaient déjà été reçus dans le SST. La question peut se poser de l'intérêt qu'il y aurait eu d'attendre le résultat du CNR avant de lancer notre démarche mais cela aurait eu l'inconvénient de rajouter encore 3 jours de latence pour des agents très impactés par la situation et souvent en demande de prise en charge. 
La possibilité de coexistence des souches tox+ et tox- chez les deux frères a finalement été écartée par le CNR car d'autres cultures obtenues à partir d'autres prélèvements chez ces derniers sont restés toujours tox-. Les deux garçons n'étaient donc pas des cas secondaires de leur sœur, mais plutôt d'autres cas «co-primaires» ayant développé une diphtérie oropharyngée asymptomatique, qui se seraient infectés sur une souche différente avec un génotype bien différent (le Multi Locus Sequence Type de la souche des deux frères (ST411) était très différent de celui isolé de la sœur (ST217) (S. Brisse et E. Badell, CNR, Comm. pers.)).

Il est important de noter que leur grande sœur, certainement cas importé du Sénégal, pays d'endémicité de la diphtérie, bien qu'à jour de sa vaccination, a contracté un germe $C d$ tox+ dans ses plaies probablement polycontaminées. Cela pourrait s'expliquer par le contexte de maladie drépanocytaire. En effet, l'état de la rate des patients atteints de cette pathologie, précocement lésée par les vaso-occlusions et saturée par l'hémolyse des hématies falciformes, permet un plus faible contrôle et un milieu plus propice au développement des infections, notamment bactériennes. On retrouve plus fréquemment chez ces patients des infections bactériennes communautaires à pneumocoque ou salmonelles (8). Les lésions cutanées des deux frères, consécutives à des piqûres de moustiques, également polycontaminées n’ont quant à elles jamais révélé la présence de Corynebacteries.

Concernant la vaccination, l'OMS dans son dernier rapport du 4 Août 2017 (3) insiste sur l'efficacité suffisante de trois doses de primo vaccination associées à trois doses de rappel dans l'enfance et l'adolescence pour maintenir une immunité contre la diphtérie durant l'enfance et l'âge adulte sans se positionner encore sur la nécessité de doses de rappel ultérieures et leur fréquence (9). Le Plan d'action mondial pour les vaccins (GVAP : Global Vaccine Action Plan) de l'OMS, a permis qu'en 2015, 86\% des nourrissons dans le monde 
(116,1 millions) reçoivent 3 doses du vaccin contre la diphtérie, le tétanos et la coqueluche (DTC) (9).

En France, une étude de 2008 (10), ayant étudié l'immunité vaccinale chez 331 sujets âgés de 18 à 60 ans recrutés dans des centres de vaccinations pour voyageurs et ayant suivi les recommandations vaccinales françaises, montre que 286 soit $86,4 \%$ avaient une concentration d'anticorps anti diphtériques > 0,1UI/mL considérée comme protectrice. Plus précisément, 97\% des personnes âgées de 18 à 29 ans avaient des anticorps à un taux protecteur, alors que seulement $62,7 \%$ des 50 à 60 ans possédaient un taux suffisant. On retrouvait un nombre de doses reçues tout au long de leur vie inférieur pour ces personnes plus âgées et un délai plus long depuis la date de la dernière injection de rappel vaccinal, ce qui s'explique parce que la recommandation de rappels diphtériques chez l'adulte est relativement récente.

L'OMS insiste sur le fait que «le principal facteur prédisposant à la maladie n'est pas la diminution de l'immunité dans le temps mais l'absence de vaccination » (9).

\section{Conclusion}

Dans ce cas clinique, à la suite d'un portage ORL de $C d$ tox- chez deux enfants, 41 professionnels contacts ont eu un prélèvement naso-pharyngé dans le $\mathrm{CHU}, 31$ ont reçu une ABP et 9 ont bénéficié d'un rappel vaccinal. Tous les prélèvements chez les professionnels sont revenus négatifs et il n’y a pas eu de cas secondaire.

Cette enquête fait suite à la prise en charge, dans l'hôpital pour adultes voisin, de la sœur de nos deux patients, infectée par une souche de $C d$ tox + avec 34 soignants impliqués dans un possible contage cutané par les plaies contaminées de la patiente. De même, 2 autres enquêtes 
récentes ont concerné : un cas de diphtérie cutanée à $C d$ tox+ chez une patiente de retour d'un voyage aux Comores, ayant entrainé la prise en charge de 9 soignants contacts, et trois mois plus tard un cas de diphtérie oropharyngée à $C d$ tox-, ayant entrainé la prise en charge de 73 soignants contacts parmi les personnels de l'hôpital. Heureusement aucun cas secondaire n'est à déplorer dans chacune de ces enquêtes.

Ces épisodes inattendus et exceptionnels rappellent l'importance du respect du calendrier vaccinal chez le personnel soignant, de la vigilance et des précautions standard et complémentaires à mettre en œuvre vis-à-vis de pathologies infectieuses devenues rares et de ce fait méconnues.

L'alerte est mise sur le manque de suivi des recommandations vaccinales, et notamment des rappels chez les personnes adultes et après 50 ans. Le vaccin antidiphtérique procure une immunité de groupe avec un seuil de $80 \%$; c'est à dire que la maladie épidémique peut réapparaître lorsque la couverture vaccinale passe au dessous de $80 \%$ de personnes vaccinées dans une population. (Le niveau d'immunité dans la population où le nombre de cas reste constant est appelé seuil d'immunité de groupe, chaque malade ne générant en moyenne qu'un seul nouveau cas car le reste de la population est bien vacciné). Le taux de reproduction (Ro) de la diphtérie est de 5, le Ro représentant le nombre moyen de personnes que contamine un sujet malade dans une population réceptive (11).

Dans cette situation n'oublions pas que la diphtérie pourrait entrainer une épidémie dramatique comme celle vécue en URSS des années 1990, atteignant 19462 cas en 1993; elle a culminé en 1994 et 1995 avec respectivement 47808 et 50412 cas. Les taux de létalité allaient de 2 à 3\% (Russie, Ukraine) à plus de 20\% (Géorgie, Azerbaïdjan, Turkménistan) (12). Et beaucoup plus récemment, fin 2017, des épidémies sont survenues au Bangladesh 
(13) et au Yémen (14) faisant encore de nos jours des centaines de contaminés et des dizaines de morts.

Pour essayer de répondre à ces problématiques, l'Organisation Mondiale de la Santé à créé le SAGE (Strategic Advisory Group of Experts on immunization) (9) afin d'uniformiser la déclaration de la diphtérie au niveau mondial, collecter les données et surveiller les flambées épidémiques. Ce groupe a récemment exprimé « une profonde préoccupation concernant les stocks limités d'anatoxine diphtérique dans le monde » (actuelle pénurie) et a souligné le besoin urgent de disposer de cette antitoxine pour prendre en charge les cas suspects de diphtérie.

Rappelons enfin qu'en tant que prévention primaire des infections, les vaccins réduisent l'usage des antibiotiques. Dans notre environnement actuel, avec l'émergence d'agents pathogènes multi résistants, les vaccins pourraient devenir la seule arme disponible pour les combattre.

Nous remercions pour leur aide et leurs conseils pour cette prise en charge : les Dr Brisse et

Dr Badell-Ocando de l'Institut Pasteur, Unité Biodiversité et Epidémiologie des Bactéries Pathogènes, Centre national de référence des Corynébactéries du complexe diphtheriae, Paris.

\section{Les auteurs déclarent ne pas avoir de conflit d'intérêt en relation avec cet article.}




\section{$\underline{\text { Références : }}$}

1. HCSP. Conduite à tenir lors de l'apparition d'un cas de diphtérie [Internet]. Paris: Haut Conseil de la Santé Publique; 2011 mars [cité 14 janv 2017]. Disponible sur: http://www.hcsp.fr/explore.cgi/avisrapportsdomaine?clefr=215

2. INRS. Corybacteries du complexe diphteriae - Agents de la Diphtérie [Internet]. 2013 [cité 14 janv 2017]. Disponible sur: http://www.inrs.fr/eficatt/eficatt.nsf/(allDocParRef)/FCDIPHTERIE?OpenDocument

3. OMS Organisation mondiale de la santé, WHO. Diphtheria vaccine: WHO position paper - August 2017. Releve Epidemiol Hebd. 04 2017;92(31):417 $\square 35$.

4. OMS Organisation Mondiale de la Santé, WHO. The immunological basis for immunization series : module 2: diphtheria - Update 2009. WHO Library Cataloguing-inPublication Data; 2009.

5. Loi du 25 juin 1938 (JO 27 juin 1938, p. 7371). Obligation de la vaccination antidiphtérique pour tous les enfants à partir de l'âge d'1 an et jusqu'à 14 ans. 1938;

6. Invs. Données épidémiologiques / Diphtérie / Maladies à déclaration obligatoire / Maladies infectieuses / Dossiers thématiques / Accueil [Internet]. invs.santepubliquefrance.fr. 2015 [cité 25 oct 2017]. Disponible sur: http://invs.santepubliquefrance.fr/Dossiers-thematiques/Maladies-infectieuses/Maladiesa-declaration-obligatoire/Diphterie/Donnees-epidemiologiques

7. Code de la santé publique - Article D3113-6 [Internet]. Code de la santé publique. Disponible sur: https://www.legifrance.gouv.fr/affichCodeArticle.do;jsessionid=94B7CFF3D18C1F9CB2 037DA74D2C69F5.tplgfr40s_3?idArticle=LEGIARTI000006911759\&cidTexte=LEGIT EXT000006072665\&categorieLien=id\&dateTexte $=20050223$

8. Inserm E Jacques. Drépanocytose [Internet]. 2014 [cité 2 janv 2018]. Disponible sur: https://www.inserm.fr/information-en-sante/dossiers-information/drepanocytose

9. OMS Organisation mondiale de la santé, WHO. Meeting of the Strategic Advisory Group of Experts on immunization, April 2017 - conclusions and recommendations. Releve Epidemiol Hebd. 02 2017;92(22):301 $\square 20$.

10. Launay $\mathrm{O}$, Toneatti $\mathrm{C}$, Bernède $\mathrm{C}$. Antibodies to tetanus, diphtheria and pertussis among healthy adults vaccinated according to the French vaccination recommendations (PDF Download Available). ResearchGate [Internet]. juin 2009 [cité 14 août 2017]; Disponible sur:

https://www.researchgate.net/publication/24019580_Antibodies_to_tetanus_diphtheria_a nd_pertussis_among_healthy_adults_vaccinated_according_to_the_French_vaccination_r ecommendations

11. Santé Publique France. Santé publique France - Dossier pédagogique - Vaccination : la protection collective [Internet]. 2017 [cité 11 avr 2018]. Disponible sur: https://www.santepubliquefrance.fr/Actualites/Vaccination-des-jeunes-enfants-des- 
donnees-pour-mieux-comprendre-1-action-publique/Dossier-pedagogique-n-2Vaccination-la-protection-collective

12. Rey M, Patey O, Vincent-Ballereau F. Retour de la diphtérie en Europe. Eurosurveillance. 1 févr 1996;1(2):14 $\square 6$.

13. Organisation Mondiale de la Santé. OMS | Propagation rapide de la diphtérie dans le district de Cox's Bazar au Bangladesh [Internet]. WHO. 2017 [cité 12 avr 2018]. Disponible sur: http://www.who.int/mediacentre/news/releases/2017/diphtheriaspreading-bangladesh/fr/

14. Organisation Mondiale de la Santé. OMS | L'OMS livre des médicaments tandis que la diphtérie se propage au Yémen [Internet]. WHO. 2017 [cité 12 avr 2018]. Disponible sur: http://www.who.int/mediacentre/news/releases/2017/medicines-diphtheria-yemen/fr/ 
Figure 1 : Historique du cas clinique

\begin{tabular}{|c|c|c|c|c|c|}
\hline $\begin{array}{l}\text { Retour du } \\
\text { Sénégal }\end{array}$ & $\begin{array}{l}\text { Passage aux } \\
\text { Urgences Z. }\end{array}$ & $\begin{array}{c}\text { Hospitalisation } \\
\text { Z. }\end{array}$ & $\begin{array}{l}\text { Diagnostic } \\
\text { diphtérie } \\
\text { cutanée Z. }\end{array}$ & $\begin{array}{l}\text { Bactérie } \\
\text { tox }+\mathrm{Z}\end{array}$ & $\begin{array}{l}\text { Lancement de la } \\
\text { démarche en SST pour } \\
\text { les contacts de I. et Y. }\end{array}$ \\
\hline 1 & & 1 & & $\perp$ & $<$ \\
\hline $\mathrm{J} 0$ & $\mathrm{~J}+3$ & $\mathrm{~J}+8$ & $\mathrm{~J}+14$ & $\mathrm{~J}+15$ & $\overbrace{}^{\mathrm{J}+18}$ \\
\hline $\begin{array}{c}\text { Passage aux } \\
\text { Urgences I. }\end{array}$ & \multicolumn{2}{|c|}{$\begin{array}{l}\text { SAU puis UHCD puis } \\
\text { hospitalisation } 2 \text { jours I. }\end{array}$} & $\begin{array}{l}\text { Hospitalisation } \\
\text { I. et Y. }\end{array}$ & $\begin{array}{l}\text { Alerte } \\
\text { du SST }\end{array}$ & $\begin{array}{c}\text { Portage ORL de } \\
\text { Cd I. et Y. }\end{array}$ \\
\hline
\end{tabular}




\section{Figure 2: Conduite à tenir en Service de Médecine et Santé au Travail pour le personnel hospitalier en cas de cas de Diphtérie à forme ORL ou cutanée}

Dès suspicion d'un cas de diphtérie : Signalement nécessaire à l'ARS par le service hospitalier Détection de tous les sujets en contact, dans les sept jours précédents, avec des sécrétions oropharyngées ou avec une lésion cutanée du cas source

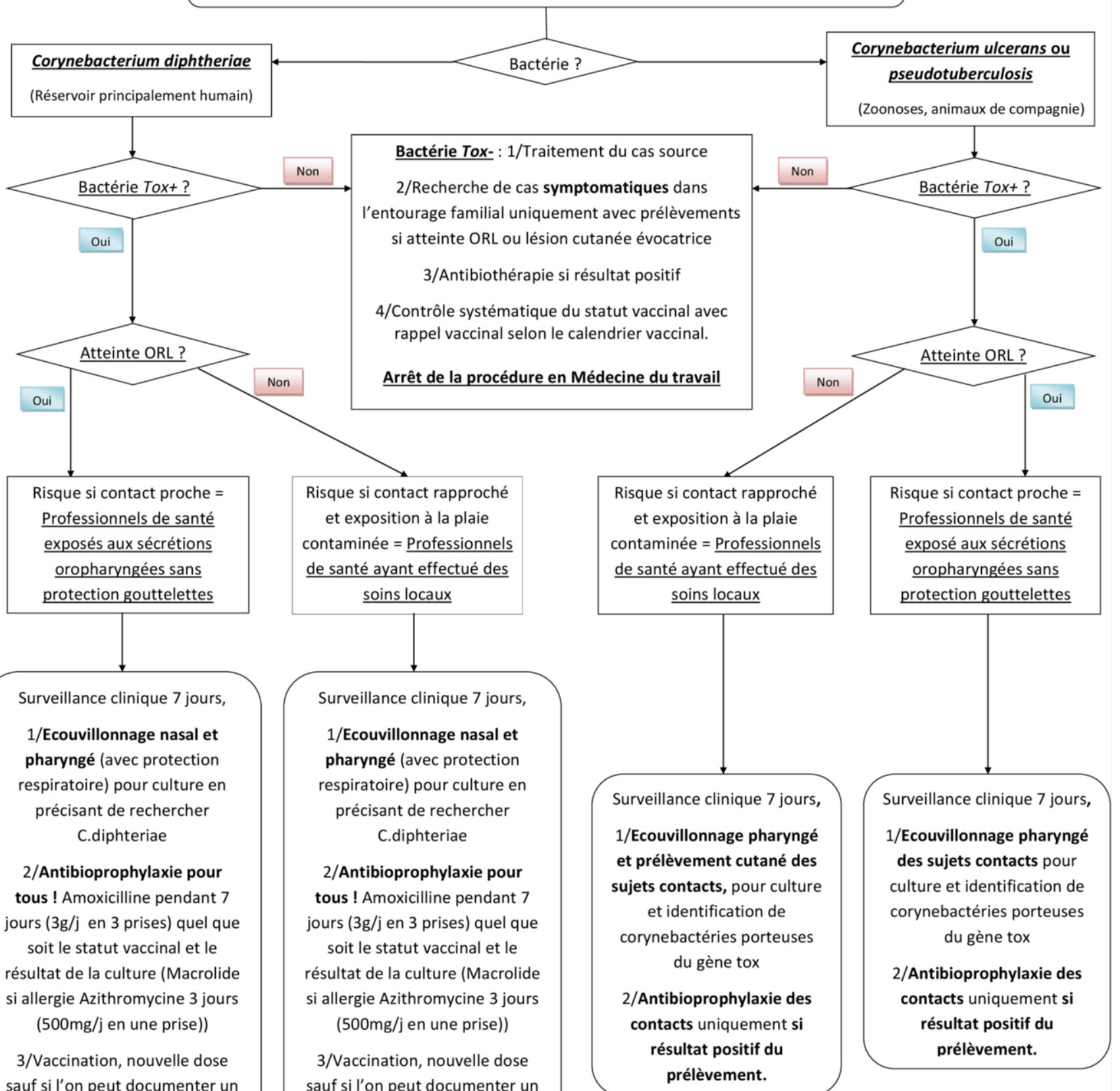

sauf si l'on peut documenter un antécédent de primovaccination d'au moins 3 doses et un dernier rappel datant de moins de 5ans.

sauf si l'on peut documenter un antécédent de primo-

vaccination d'au moins 3 doses et un dernier rappel datant de moins de 5 ans. 
Figure 3 : Flow chart de la population

78 personnels de l'hôpital contactés

14 n'avaient pas réellement été en contact avec les deux enfants
64 ont donc été définis comme sujets contacts

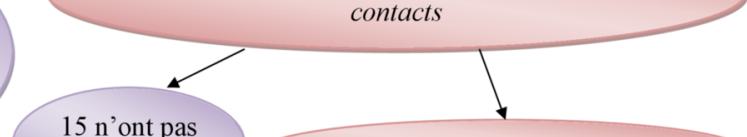

15 n'ont pas répondu
49 ont donné suite à l'alerte

3 médecins/internes avaient pris toutes les précautions d'isolement nécessaires

5 médecins/internes ont été pris en charge dans un autre établissement

41 personnes ont eu des écouvillonnages nasaux et pharyngés dans le $\mathrm{CHU}$

2 agents du transport externe ont été adressés à leur hôpital de ralliement
38 personnes par le SST de l'hôpital et 1 dans son service 
Figure 4 : Descriptif des 38 agents prélevés au Service de Santé au Travail et une dans son service

\begin{tabular}{|c|c|c|c|c|c|c|}
\hline Service & Sexe & Fonction & $\begin{array}{c}\text { Ecouvillonnages } \\
\text { nasaux et pharyngés }\end{array}$ & Résultat & ATB & $\begin{array}{c}\text { Rappel } \\
\text { vaccinal }\end{array}$ \\
\hline \multirow{13}{*}{$\begin{array}{c}\text { Service } \\
\text { d'Accueil des } \\
\text { Urgences } \\
\text { (2 AP, } \\
10 \text { IDE et } 1 \\
\text { interne) }\end{array}$} & $\mathrm{F}$ & AP & OUI & NEGATIF & OUI & OUI \\
\hline & $\mathrm{F}$ & $\mathrm{AP}$ & OUI & NEGATIF & OUI & NON \\
\hline & $\mathrm{F}$ & IDE & OUI & NEGATIF & NON & NON \\
\hline & $\mathrm{F}$ & IDE & OUI & NEGATIF & OUI & $\mathrm{NON}$ \\
\hline & $\mathrm{F}$ & IDE & OUI & NEGATIF & OUI & $\mathrm{NON}$ \\
\hline & $\mathrm{F}$ & IDE & $\begin{array}{l}\text { OUI faits dans son } \\
\text { service }\end{array}$ & NEGATIF & OUI & NON \\
\hline & $\mathrm{H}$ & IDE & OUI & NEGATIF & OUI & $\mathrm{NON}$ \\
\hline & $\mathrm{F}$ & IDE & OUI & NEGATIF & OUI & $\mathrm{NON}$ \\
\hline & $\mathrm{F}$ & IDE & OUI & NEGATIF & OUI & $\mathrm{NON}$ \\
\hline & $\mathrm{F}$ & IDE & $\begin{array}{c}\text { OUI refaits après } \\
\text { passage aux urgences }\end{array}$ & NEGATIF & OUI & OUI \\
\hline & $\mathrm{F}$ & IDE & OUI & NEGATIF & NON & $\mathrm{NON}$ \\
\hline & $\mathrm{F}$ & IDE & OUI & NEGATIF & $\mathrm{NON}$ & OUI \\
\hline & $\mathrm{F}$ & Interne & OUI & NEGATIF & OUI & $\mathrm{NON}$ \\
\hline \multirow{6}{*}{$\begin{array}{c}\text { Unité } \\
\text { d'Hospitali- } \\
\text { sation de } \\
\text { Courte Durée } \\
\text { (2 AP et } \\
4 \text { IDE) }\end{array}$} & $\mathrm{F}$ & Etudiant AP & OUI & $\begin{array}{c}\text { NON } \\
\text { VALIDE }\end{array}$ & OUI & $\mathrm{NON}$ \\
\hline & $\mathrm{F}$ & $\mathrm{AP}$ & OUI & NEGATIF & OUI & NON \\
\hline & $\mathrm{H}$ & Etudiant IDE & OUI & NEGATIF & OUI & NON \\
\hline & $\mathrm{F}$ & IDE & $\begin{array}{c}\text { OUI refaits après } \\
\text { passage aux urgences }\end{array}$ & NEGATIF & OUI & NON \\
\hline & $\mathrm{F}$ & IDE & OUI & NEGATIF & OUI & $\mathrm{NON}$ \\
\hline & $\mathrm{F}$ & IDE & OUI & NEGATIF & OUI & OUI \\
\hline \multirow{3}{*}{$\begin{array}{l}\text { Radiologie } \\
\text { (3 agents) }\end{array}$} & $\mathrm{F}$ & Manipulateur & OUI & NEGATIF & OUI & OUI \\
\hline & $\mathrm{F}$ & Manipulateur & OUI & NEGATIF & OUI & $\mathrm{NON}$ \\
\hline & $\mathrm{F}$ & Manipulateur & OUI & NEGATIF & OUI & NON \\
\hline Brancardage & $\mathrm{F}$ & Brancardier & OUI & NEGATIF & OUI & $\mathrm{NON}$ \\
\hline \multirow{13}{*}{$\begin{array}{c}\text { Centre } \\
\text { spécialisé en } \\
\text { hématologie } \\
\text { pédiatrique } \\
\text { (3 AP, } 6 \mathrm{IDE} \\
\text { et } 4 \mathrm{ASH} \text { ) }\end{array}$} & $\mathrm{F}$ & $\mathrm{AP}$ & OUI & NEGATIF & OUI & $\mathrm{NON}$ \\
\hline & $\mathrm{F}$ & AP & OUI & NEGATIF & OUI & NON \\
\hline & $\mathrm{F}$ & $\mathrm{AP}$ & OUI & NEGATIF & OUI & $\mathrm{NON}$ \\
\hline & $\mathrm{F}$ & IDE & OUI & NEGATIF & NON & OUI \\
\hline & $\mathrm{F}$ & IDE & OUI & NEGATIF & NON & $\mathrm{NON}$ \\
\hline & $\mathrm{F}$ & IDE & OUI & NEGATIF & OUI & $\mathrm{NON}$ \\
\hline & $\mathrm{F}$ & IDE & OUI & NEGATIF & OUI & OUI \\
\hline & $\mathrm{F}$ & IDE & OUI & NEGATIF & OUI & NON \\
\hline & $\mathrm{F}$ & IDE & OUI & NEGATIF & $\mathrm{NON}$ & $\mathrm{NON}$ \\
\hline & $\mathrm{F}$ & ASH & OUI & NEGATIF & $\mathrm{NON}$ & $\mathrm{NON}$ \\
\hline & $\mathrm{F}$ & ASH & OUI & NEGATIF & $\mathrm{NON}$ & NON \\
\hline & $\mathrm{F}$ & ASH & OUI & NEGATIF & $\mathrm{NON}$ & NON \\
\hline & $\mathrm{F}$ & $\mathrm{ASH}$ & OUI & NEGATIF & OUI & OUI \\
\hline \multirow{3}{*}{$\begin{array}{c}\text { Services } \\
\text { administratifs } \\
\text { (3 agents) }\end{array}$} & $\mathrm{F}$ & $\begin{array}{l}\text { Secrétaire } \\
\text { médicale }\end{array}$ & OUI & NEGATIF & OUI & $\mathrm{NON}$ \\
\hline & $\mathrm{F}$ & $\begin{array}{l}\text { Secrétaire } \\
\text { médicale }\end{array}$ & OUI & NEGATIF & NON & $\mathrm{NON}$ \\
\hline & $\mathrm{F}$ & $\begin{array}{l}\text { Secrétaire } \\
\text { médicale }\end{array}$ & OUI & NEGATIF & OUI & OUI \\
\hline
\end{tabular}

IDE : Infirmier Diplômé d'Etat/AP : Auxiliaire de Puériculture/ASH : Aide Sanitaire Hospitalier 